\title{
The Examination on Corneal Nerve in Diabetic WBN/Kob Rat is Useful for Detecting Peripheral Sensory Neuropathy
}

\author{
Kiyokazu Ozaki ${ }^{1}$, Yui Terayama ${ }^{1}$ and Tetsuro Matsuura ${ }^{1}$ \\ 1. Laboratory of Pathology, Faculty of Pharmaceutical Science, Setsunan University, Hirakata, Osaka, \\ Japan \\ * Corresponding author: ozaki@pharm.setsunan.ac.jp
}

Corneal confocal microscopy (CCM) has been used to evaluate small nerve fibers in the cornea [1]. CCM studies also have revealed that the density of nerve fibers in the subbasal nerve plexus (SBNP) correlates with the severity of diabetic sensory neuropathy in human patients, suggesting that such analyses represent an alternative, noninvasive marker of peripheral neuropathy in patients with diabetes. As for animal models, some researchers have investigated the severity of diabetic peripheral neuropathy by evaluating the corneal nerves within the SBNP in mouse and rat models of diabetes [2]. However, such studies have showed contradictory results, with some demonstrating significant decreases in fiber density and others reporting no abnormalities. Recently, we have elucidated that extended duration of hyperglycemia result in human-like corneal nerve lesions in mice with alloxan- and streptozotocin-induced type 1 diabetes [3]. WBN/Kob rats spontaneously develop long-lasting diabetes and human-like diabetic peripheral motor neuropathy characterized by segmental demyelination and axonal atrophy, with slowing of the nerve conduction velocity $[4,5]$. However, morphological change of the sensory nerve terminal remains to be elucidated in this strain. In the present study, we investigated whether the density of corneal nerve fiber can be useful as an alternative morphological marker of diabetic peripheral neuropathy in diabetic $\mathrm{WBN} / \mathrm{Kob}$ rats.

Male diabetic WBN/Kob rats were used along with age-matched non-diabetic females. Male rats aged 36 weeks showed early diabetes and males 90 aged weeks showed long-lasting diabetes, respectively. Paraformaldehyde-fixed corneas were excised and made transparent via immersion in optical cleaning agent. The cornea was immuno-stained with tubulin $\beta 3$ antibody. Z-stack images of nerve fibers were taken in the central and peripheral corneal region using a confocal microscope. The percent area of the terminal nerve fibers in the epithelial area (terminal epithelial nerve density [TEND]) and the percent area of nerve fibers in the sub-basal area (sub-basal nerve plexus density [SBNPD]) were determined.

SBNPD and TEND in the central cornea were significantly increased with ageing in non-diabetic females aged 90 weeks compared to those of non-diabetic females aged 36 weeks. However, in male rats, the increase of SBNPD and TEND in the central cornea was prevented by progression of diabetes, and those aged 90 weeks were markedly decreased as much as $40 \%$ compared to females aged 90 weeks. SBNPD of the peripheral cornea in male aged 90 weeks was decreased as much as $40 \%$ compared to females aged 90 weeks, showing no difference between them in the central cornea.

These results indicate that the examination on corneal nerve (TEND and SBNTD) in spontaneously diabetic WBN/Kob rats can be useful for detecting diabetic peripheral sensory neuropathy.

References:

[1] I Ostrovski et al., PLoS One 10 (2015), p. e0142309. 
[2] D Cai et al., Am J Pathol 184 (2014), p. 2662.

[3] K Ozaki, Y Terayama and T Matsuura, Invest Ophthalmol Vis Sci 59 (2018), p. 5868.

[4] K Ozaki et al., Acta Neuropathol (Berl) 92 (1996), p. 603.

[5] K Ozaki et al., J Vet Med Sci 75 (2013), p. 1323.

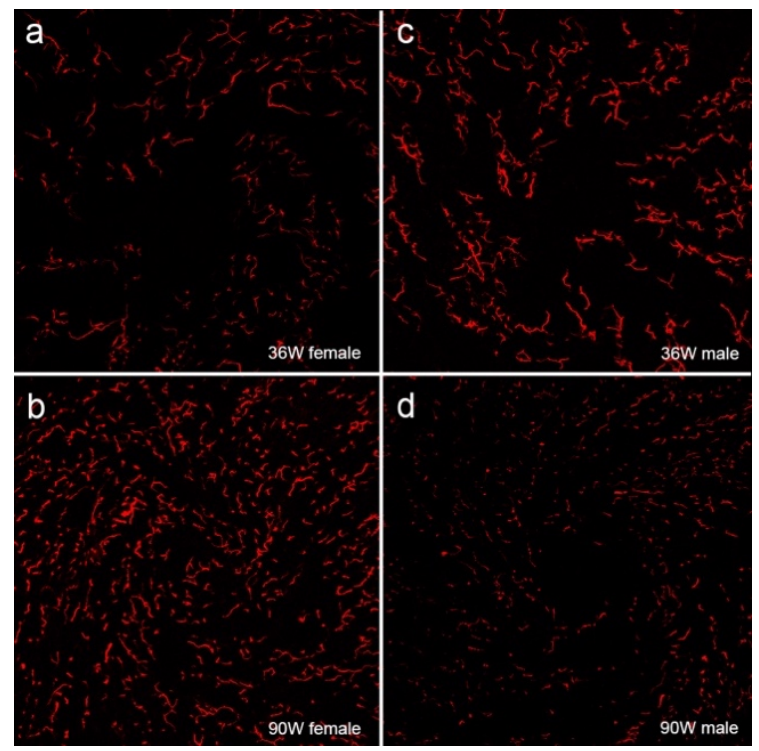

Figure 1. Tubulin $\beta 3$ positive-nerve fibers in terminal epithelial nerves (TENs) of the corneal epithelium in the central cornea. TEND was significantly increased in non-diabetic females aged 90 weeks compared to those of non-diabetic females aged 36 weeks. However, in male rats, the increase of TEND in the central cornea was prevented by progression of diabetes.

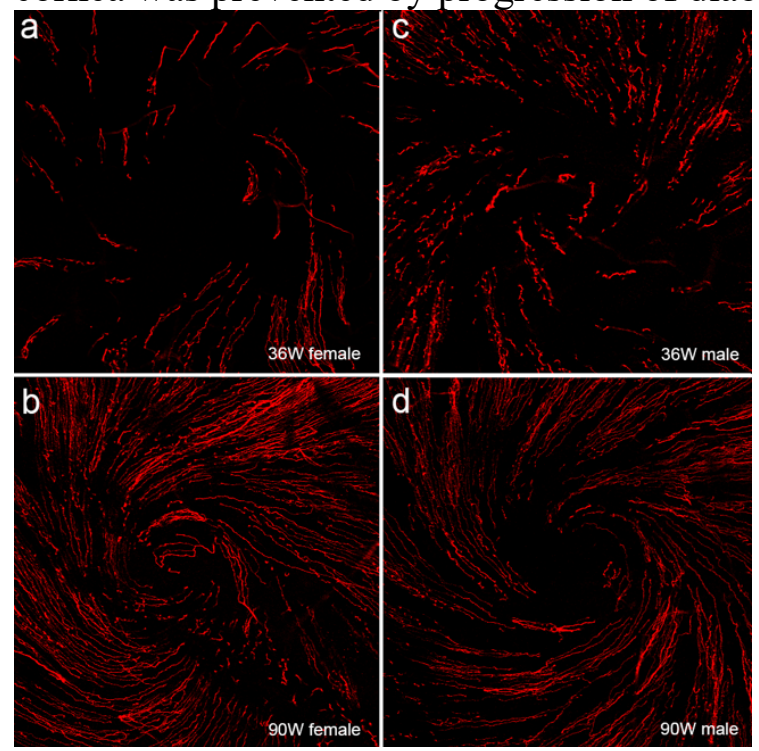

Figure 2. Tubulin $\beta 3$ positive-nerve fibers in the sub-basal nerve plexus (SBNP) of the central cornea. SBNPD was significantly increased in non-diabetic females aged 90 weeks compared to those of nondiabetic females aged 36 weeks. However, in male rats, the increase of SBNPD in the central cornea was prevented by progression of diabetes. 